\title{
Nanogold synthesis in wool fibres: novel colourants
}

\author{
James H. Johnston • Kerstin A. Lucas
}

Published online: 9 April 2011

(C) The Author(s) 2011. This article is published with open access at Springerlink.com

\begin{abstract}
This paper presents the novel synthesis of gold nanoparticles of different sizes and hence colours in a wool fibre matrix, simultaneously utilising the chemical affinity of gold for sulfur to bind the nanogold to the disulfide linkages in cystine amino acids in the keratin protein. For this, the wool fibres act as a solid matrix to control the particle size and prevent agglomeration of the gold nanoparticles and hence facilitate a range of attractive colours in the wool due to the surface plasmon resonance effects of such gold nanoparticles. Because the nanogold is chemically bound to the cystine, it does not wash or rub out and is also stable to UV light, unlike organic colourants. The research innovatively links the high value and prestige of gold through nanoscience for high value textiles and fashion apparel, wherein the nanogold wool composite fibres contain only pure wool and pure gold and are environmentally desirable.
\end{abstract}

Keywords Nanogold $\cdot$ Wool $\cdot$ Colourant, Textile, Keratin protein

\section{Introduction}

Gold has long been known to form stable colloids of nanosize particles. Such nanoparticles exhibit different

\footnotetext{
J. H. Johnston $(\square) \cdot$ K. A. Lucas

School of Chemical and Physical Sciences and the MacDiarmid Institute for Advanced Materials and Nanotechnology,

Victoria University of Wellington,

P.O. Box 600, Wellington, New Zealand

e-mail: jim.johnston@vuw.ac.nz
}

colours due to surface plasmon resonance effects which result from the resonance interaction of incoming electromagnetic radiation in the visible region with the collective plasmon oscillations at the metal surface [1]. This gives rise to intense absorptions in the visible spectrum. The colour is dependent on the particle size and shape and the dielectric constant of the surrounding medium. Colloids of spherical nanogold particles of about $10-20 \mathrm{~nm}$ in size are red. As the particle size increases, the colour changes through darker shades of red to purple, then to blue-grey for particles or agglomerates up to about $80-100 \mathrm{~nm}$. The colours relate to a shift and broadening of the transverse plasmon resonance absorption band, originally about $520 \mathrm{~nm}$. Non-spherical gold nanoparticles also exhibit different colours depending upon their shape and aspect ratio, as they show a longitudinal plasmon resonance band at about $720 \mathrm{~nm}$ along with the transverse band [2-4]. Short gold nanorods are typically blue in colour. The change in shape from rods to dumbbell or phi-shaped particles leads to a shift in the longitudinal plasmon band to the blue and red directions respectively [4]. All the colours are stable in sunlight and UV light and do not change providing there is no change in particle size through the growth of discrete nanoparticles or agglomeration.

Interestingly, colloidal gold nanoparticles have been unknowingly used as colourants in ancient times. The Lycurgus Cup dating back to around 400 AD used gold and silver nanoparticles as colourants to provide an interesting dichroic effect in the glass where it appears red in transmitted light and green in reflected light [5]. Also, gold was used to produce rose coloured glass dating back to about the seventeenth century. In 1856, Michael 


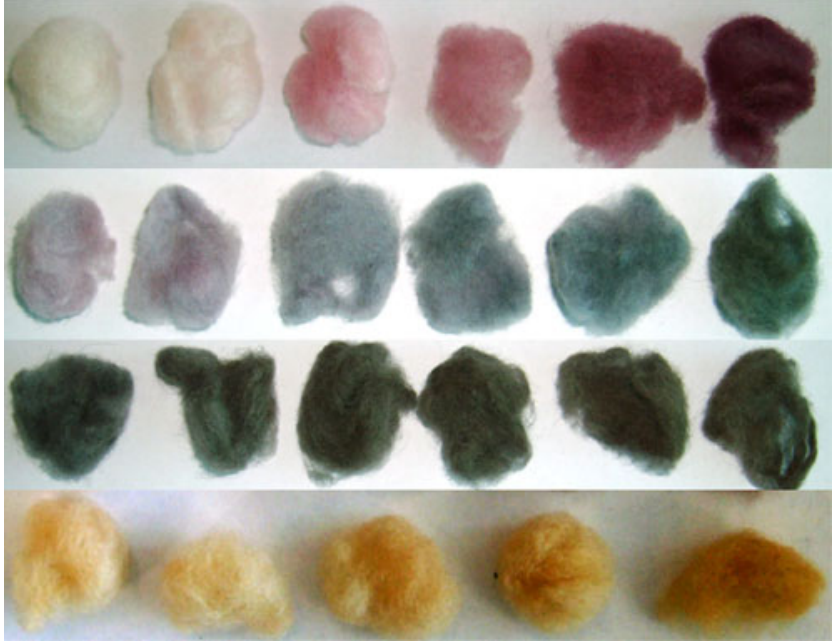

Fig. 1 A representative range of merino wool fibres coloured with gold nanoparticles of different particle sizes

Faraday was the first to recognize that it was the colloidal gold which acted as the colourant in the glass. Following this in 1908, Mie [6] provided a theoretical explanation by solving Maxwell's equations for the absorption and scattering of electromagnetic radiation by very small metallic particles, i.e. nanoparticles.

Wool fibres have been used since historical times in apparel, furnishings and carpet where the inherent properties of flexibility, crimp, porosity and water resistance are important attributes that advantageously distinguish them from synthetic fibres.

Merino wool with its smaller fibre diameter in the range 12-20 microns is highly valued for fine apparel whereas strong wools of 25-40 microns in diameter are desired for harder wearing furnishing textiles and carpet. Keratin is a principal protein in wool with the sulfur-containing amino acid cystine, which provides a possible binding site for nanogold via the disulfide linkage, due to the chemical affinity of gold for sulfur.

We have captured the unique opportunity to utilise the intense visible absorption and the range of stable colours of gold nanoparticles as novel colourants for wool, thereby linking the premier position and high value of gold in society with the nanoscience of gold, to provide a range of nanogold coloured wools for luxury apparel and furnishing textiles and carpet [7].

\section{Nanogold wool}

Gold nanoparticles can be readily synthesised by treatment of an $\mathrm{Au}^{3+}$ containing solution, usually as the $\mathrm{AuCl}_{4}{ }^{-}$ complex with a reducing agent such a tri-sodium citrate [2, 3] or monomeric and polymeric amines which act as both a reductant and a stabilizer [8]. If tri-sodium citrate is used in excess amounts, it can also act as a stabilizer. The colour of the resulting gold colloid depends on the size and shape of the gold nanoparticles which is controlled by the $\mathrm{Au}^{3+}$ concentration, the reductant type and concentration, and the stabilizer.

We have used the porous nature of wool and the affinity of gold for sulfur contained in the cystine amino acids to control the size of the gold nanoparticles and chemically bind them to the surface and within the wool fibre matrix to provide a range of nanogold wool materials in attractive colours (Fig. 1) [7]. We believe this is the first time nanogold has been synthesised and used in this way, although gold nanoparticles have been attached to silk fibres [2].

The SEM images (Fig. 2) show gold nanoparticles formed in this way and bound to the wool are sparsely scattered on the surface of the wool fibre with a higher concentration around the cuticle edges where the chemical potential is higher. This wool is a deep purple colour even
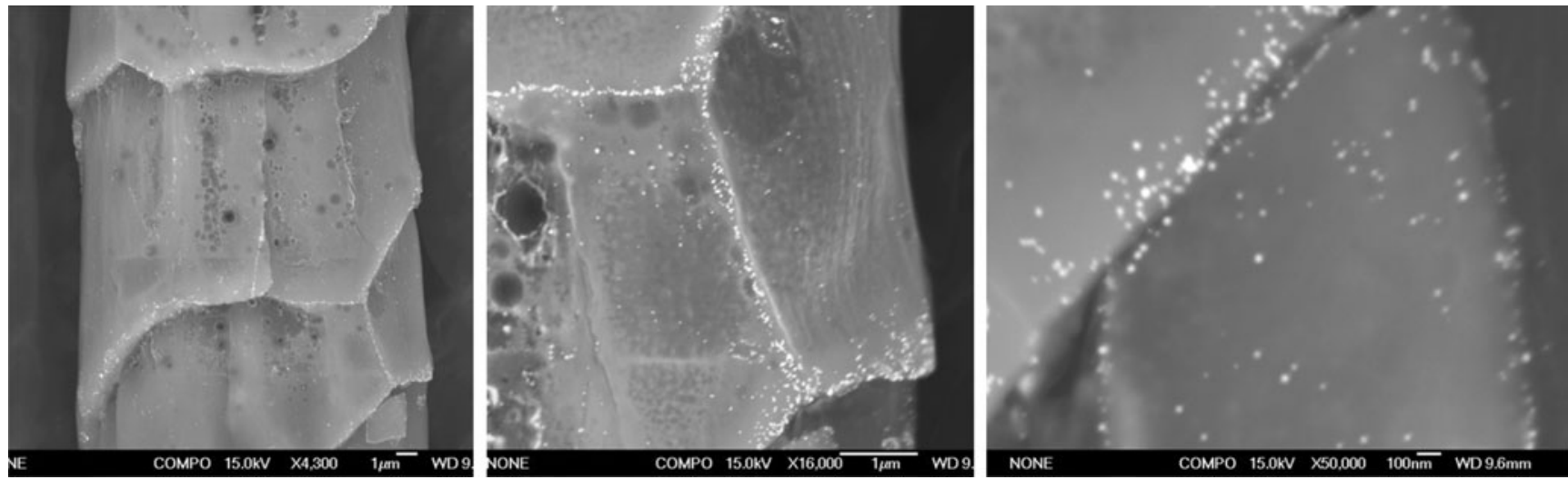

Fig. 2 SEM images at increasing magnification (left to right) of a nanogold wool fibre showing gold nanoparticles (white dots) on the surface of the fibre, particularly along the cuticle edges 

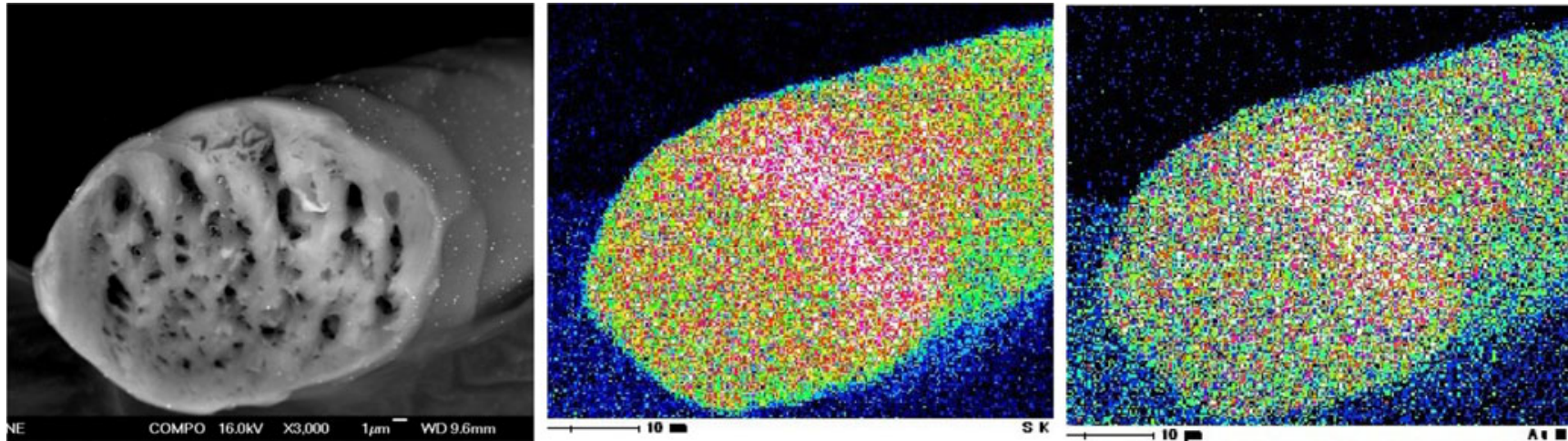

Fig. 3 SEM image of a nanogold-wool fibre showing the cross section and part of the fibre surface with the sparsely distributed gold nanoparticles just visible as white dots on the fibre surface (left). EDS

though the gold nanoparticles cover only a very small fraction of the wool surface. This is because of the very high extinction coefficient of gold under surface plasmon resonance conditions. A SEM image of a cross section of the nanogold wool fibre together with the associated EDS elemental maps for $\mathrm{Au}$ and $\mathrm{S}$ show a strong correlation between these elements, thus confirming the role of sulfur in binding the gold nanoparticles onto the surface and within the wool fibre (Fig. 3). Higher resolution TEM images (Fig. 4) of a thin cross section of the nanogold wool fibre show the gold nanoparticles on the surface are spherical and about $25-30 \mathrm{~nm}$ in size and those within the bulk fibre are about $15 \mathrm{~nm}$ in size. The growth of the gold nanoparticles on the surface is therefore greater than in the bulk. This is because the diffusion of the $\mathrm{AuCl}_{4}^{-}$ions from the surrounding solution into the wool fibre to the nanoparticle sites, where the reduction of the gold takes elemental distribution of $\mathrm{S}$ (centre) and $\mathrm{Au}$ (right). (Note: white highest concentration then purple, then green)

place and growth occurs, is slower than that on the fibre surface where the $\mathrm{AuCl}_{4}{ }^{-}$ions are in direct contact with the surface gold nanoparticles. These TEM images also show the sparse nature of the gold nanoparticles illustrating that only low levels of gold are needed to provide the range of colours for nanogold wool reported here. The variable structure and porosity of wool as a natural fibre means that the diffusion path length and rate of diffusion of the $\mathrm{AuCl}_{4}{ }^{-}$ions to a particular nanogold nucleation site will vary. Therefore, the wool matrix is not able to provide the same level of control of the particle size as is achievable in a conventional solution synthesis of gold nanoparticles. Hence, a range of particle sizes is observed (Fig. 3). However, using the wool as a solid state matrix for the gold nanoparticle formation, it is still possible to effect sufficient control to enable different colours to be produced repeatably.
Fig. 4 High resolution TEM images of a cross section of a purple nanogold wool fibre showing: (top left) the gold nanoparticles at the fibre surface (larger black spheres) and sparsely distributed throughout the bulk fibre (smaller spheres), also shown at bottom left; (top right) a higher magnification image of the gold nanoparticles at the fibre surface; (bottom right) a higher magnification image of the gold nanoparticles in the bulk fibre
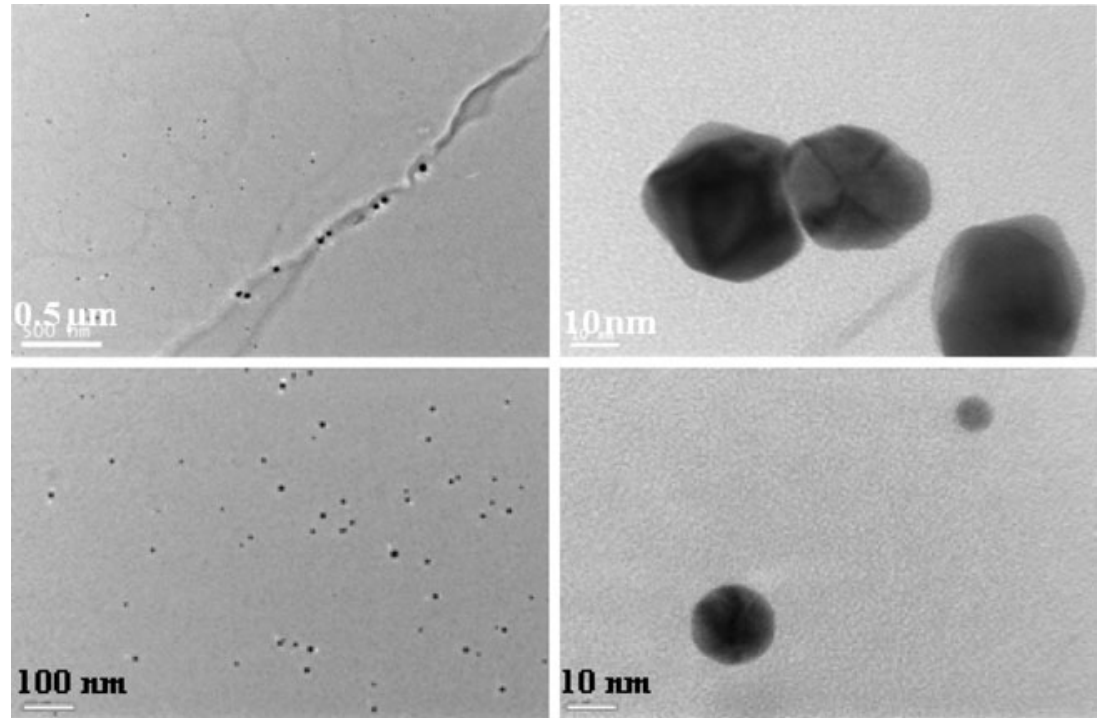

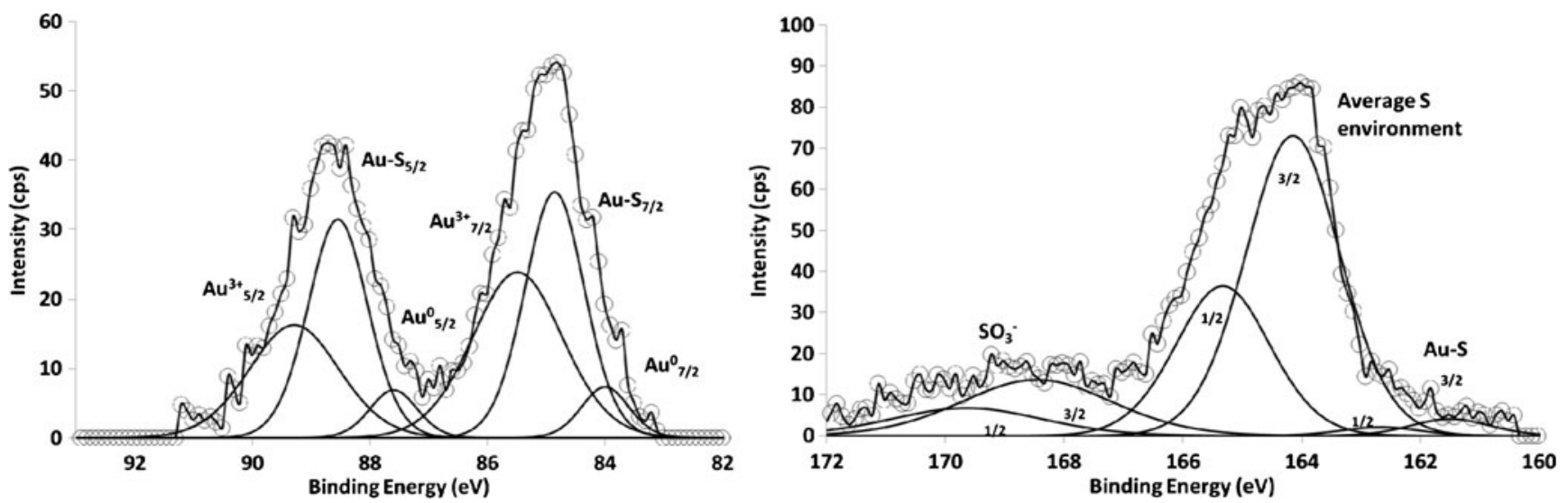

Fig. 5 High resolution XPS spectra of a nanogold wool fibre showing the experimental curve and the deconvoluted peaks for Au $4 f(l e f t)$ and $\mathrm{S}$ $2 p$ electrons (right)

The deconvoluted high resolution X-ray photoelectron spectroscopy (XPS) spectrum of the $7 / 2$ and $5 / 2 \mathrm{Au} 4 f$ electrons in the nanogold wool shows peaks that can be assigned to $\mathrm{Au}^{0}, \mathrm{Au}^{3+}$, or $\mathrm{Au}^{+}$in $\mathrm{AuS}$ (Fig. 5) [9, 10]. It is likely the $\mathrm{Au}^{3+}$ or $\mathrm{Au}^{+}$peak is due to a layer of unreduced gold on the surface of the $\mathrm{Au}^{0}$ nanoparticles and also possibly to some $\mathrm{Au}^{3+}\left(\mathrm{AuCl}_{4}{ }^{-}\right)$in the pores of the wool fibre. The AuS peak confirms the gold nanoparticles are chemically bound to the sulfur in the cystine amino acids in the keratin protein. The comparable deconvoluted high resolution XPS spectrum of the S $2 p$ electrons for sulfur in the nanogold wool fibres (Fig. 5) shows the typical $3 / 2$ and $1 / 2$ peaks for sulfur consistent with the $-\mathrm{S}-\mathrm{S}-$ disulfide entities in cystine. A small AuS peak is observable which is much less intense than the main sulfur peaks as the mol.\% of Au in the nanogold wool is correspondingly much less than the mol.\% of S [9]. There is also a peak due to oxidized sulfur, probably cysteic acid, which can arise from some natural oxidation of the cystine sulfur and also from the coupled redox reaction between the cystine $-\mathrm{S}-\mathrm{S}-$ groups which are oxidized as the $\mathrm{Au}^{3+}$ is reduced to form nanoparticulate $\mathrm{Au}^{0}[7]$.
Taking account of the above experimental evidence, a schematic model for the formation of nanogold wool can be developed. For this, the first stage is the adsorption of $\mathrm{Au}^{3+}\left(\mathrm{AuCl}_{4}^{-}\right)$ions onto the wool fibre surface and their diffusion into the fibre due to its porous nature. Because of the strong chemical affinity of gold for sulfur, it is likely that clusters of $\mathrm{Au}^{3+}\left(\mathrm{AuCl}_{4}{ }^{-}\right)$ions form in the vicinity of the $-\mathrm{S}-\mathrm{S}-$ units in the cystine amino acids of the keratin. Here, the $\mathrm{Au}^{3+}$ is reduced to $\mathrm{Au}^{0}$ and the resulting gold nanoparticles then chemically bind to the sulfur in the remaining $-\mathrm{S}-\mathrm{S}$ - cystine linkages to form a stable nanogold wool fibre. Some $\mathrm{Au}^{+}$remains on the surface of the $\mathrm{Au}^{0}$ gold nanoparticles which form the $\mathrm{Au}-\mathrm{S}$ bond. A schematic of this is shown in Fig. 6.

The nanogold wool fibres so formed were tested for lightfastness and colourfastness according to the respective standard procedures [11]. As expected, because the colour is generated by surface plasmon resonance effects and not by organic chromophores, these performed very well indeed and were superior to conventional dyes. Excellent rubfastness test results confirmed the chemical binding of the gold to the wool and the robustness of the
Fig. 6 Schematic diagram showing the formation of nanoclusters of $\mathrm{Au}^{3+}$ and their subsequent reduction to nanoparticulate $\mathrm{Au}^{0}$

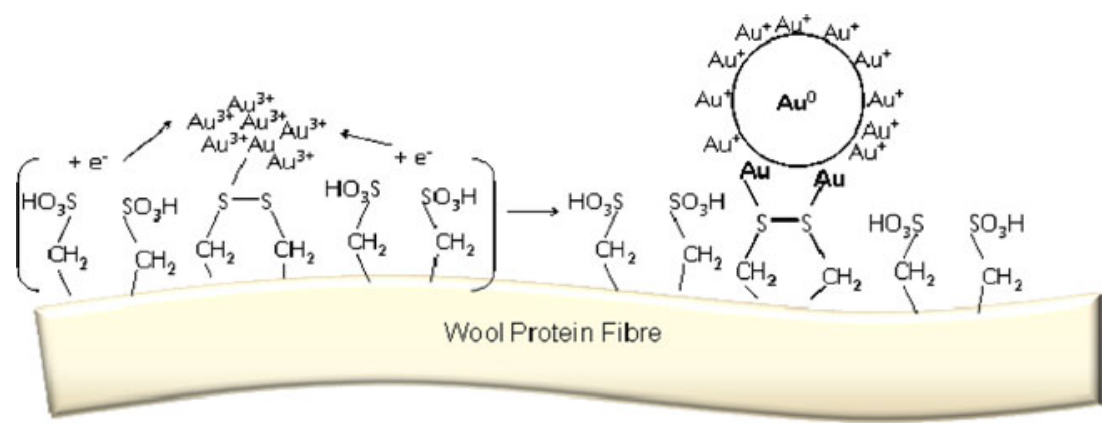



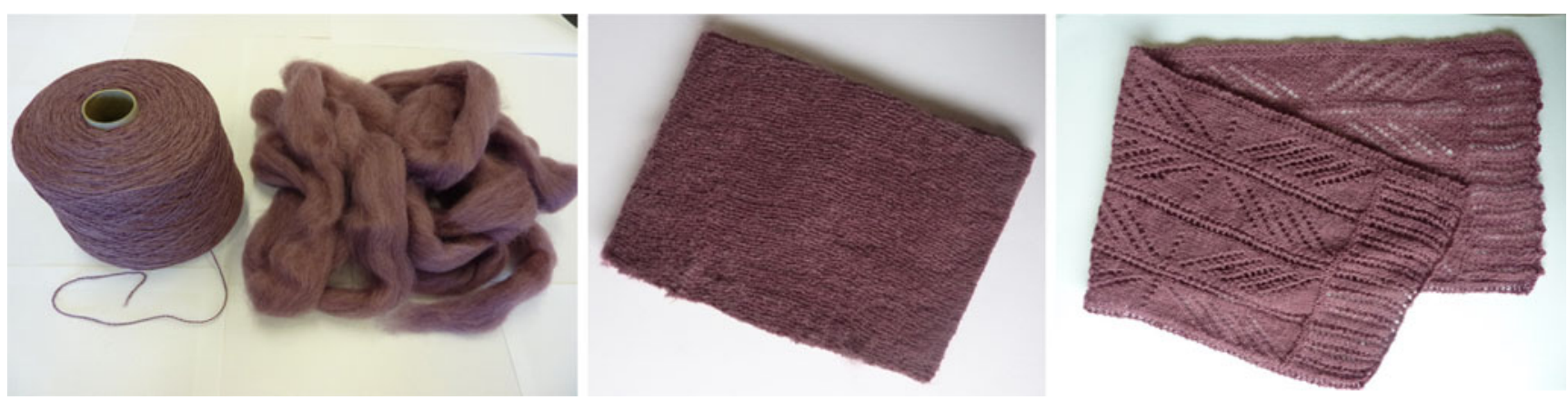

Fig. 7 A selection of purple nanogold wool products: top wool and yarn (left), carpet (centre), and merino wool scarf (right)

product. Following laboratory scale development, the nanotechnology has been successfully scaled up to colour larger quantities of wool which have then been spun into yarn and fashioned into textiles, scarves, and carpet samples (Fig. 7). The proprietary nanotechnology has been protected [7] and is being progressed to commercialization as a unique, world first nanogold wool fibre and product suite.

In summary, this paper presents an innovative approach to the synthesis and use of gold nanoparticles as novel stable colourants utilising the surface plasmon resonance colouring effect of nanogold.

Open Access This article is distributed under the terms of the Creative Commons Attribution License which permits any use, distribution and reproduction in any medium, provided the original author(s) and source are credited.

\section{References}

1. Norman TJ Jr, Grant CD, Magana D, Zhang JZ, (2002) J Phys Chem B 106:7005-7012

2. Daniel M-C, Astruc D (2004) Chem Rev 104:293-346

3. Grzelczak M, Pérez-Juste J, Mulvaney P, Liz-Marzán LM (2008) Chem Soc Rev 2(37):1783

4. Xu X, Cortie MB (2006) Adv Funct Mater 16:2170

5. Freestone I, Meeks N, Sax M, Higgitt C (2007) Gold Bulletin 40(4):270

6. Mie G (1908) Ann Phys 25:377

7. Johnston, J. H., Burridge, K. A., Kelly, F. M., Small A. C. (2010) NZ Patent Application 589498

8. Richardson MJ, Johnston JH (2007) J Colloid Interface Sci 310(2):425

9. J. Moulder, W. Stickle, P. Sobol, K. Bomben (1995) Handbook of $\mathrm{X}$-ray photoelectron spectroscopy: a reference book of standard spectra for identification and interpretation of XPS data (ed. J. Chastain). Physical Electronics: Eden Prairie, MN

10. Dodero G, Michieli LD, Cavalleri O, Rolandi R, Oliveri L, Dacca A, Parodi R (2000) Colloids Surf A Physiochemical Eng Aspects 175:121

11. Australian / NZ Standard 2111.19.2 (1996) 